\title{
Enhancing the BioSense Platform: Findings from an ESSENCE and SAS Pilot Project
}

\author{
Cassandra N. Davis* \\ Division of Health Informatics and Surveillance, Centers for Disease Control and Prevention, Atlanta, GA, USA
}

\section{Objective}

To describe the results of a pilot project that examined selected BioSense 2.0 data processing rules and tested SAS and ESSENCE products in the BioSense platform.

\section{Introduction}

BioSense was launched in 2003 by CDC with its primary aim to establish an integrated system of nationwide public health surveillance for the early detection and prompt assessment of potential bioterrorism-related syndromes or other public health emergencies. With the release of CDC's Surveillance Strategy, BioSense evolved into the National Syndromic Surveillance Program (NSSP). To overcome the challenges experienced throughout the integration of local and state level data to produce a real-time national all-hazards surveillance, CDC sought input from the National Syndromic Surveillance Community of Practice (NSSP CoP). They requested that $\mathrm{CDC}$ provide advanced syndromic surveillance functionalities and analytical applications, such as ESSENCE and SAS to improve the BioSense Platform. In response, CDC led this pilot project to: 1) conduct security testing of SAS[1] and ESSENCE[2] in order to identify vulnerabilities; 2) test and improve a limited set of processes that occur before data are transformed; and 3) conduct testing of ESSENCE's functions to ensure the tool worked as intended, and that it will meet user needs.

\section{Methods}

The pilot project was initiated on December 3, 2014 and concluded on May 15, 2015 with a proposed course of action for review by CDC's Division of Health Informatics and Surveillance and the NSSP CoP.

CDC engaged eight jurisdictions and two federal agencies via weekly calls and project activities. Each week, jurisdictions provided feedback and recommendations on outputs generated by CDC from analyzing the pre-locker processing, including facility table master clean-up, data element profiling, data landscape and flow, documenting current rules, and collapsing of records into visits. Simultaneously, installation and vulnerability scanning of SAS and ESSENCE took place.

Once complete, jurisdictions assessed ESSENCE's functions, data sharing and permissions, usability, timeliness, and ESSENCE user satisfaction. Each jurisdiction self-identified as novice, intermediate, or advanced user based on their ESSENCE experience. Data views and data sharing permissions were preset by CDC. Users were provided with a use case that included real-world tasks to test different system features. Users recorded their answers on an online survey and follow-up in-depth interviews were conducted.

\section{Results}

There were several major outcomes. ESSENCE successfully completed vulnerability scanning, and results indicated that it has many strengths. Users, overall, had a good experience using the tool and it provided flexibility to streamline data workflows, customize data views, share analyses with others, and generally meet the functional needs of users. We identified the data processing flow and components of the locker including the rules and codes that send data to different tables, views and transport mechanisms. The users also identified different segments needed to populate a few key data fields. Lastly, we identified the specific fields that should be included in the master facility list.

While there were many strengths, some weaknesses were also identified. SAS had three occurrences of high vulnerability that needed remediation. ESSENCE has no data sharing control for local administrators, data sharing and data source identification were not intuitive, the user interface did not explicitly provide information about navigating through screens, and there was a learning curve for new and intermediate users.

\section{Conclusions}

Overall, the pilot proved successful in providing suggestions for specific actions. These include data clean-up (ensuring all priority data fields are processed and stored properly), developing a new data staging environment, developing a local administration tool for data access control for ESSENCE, alpha and beta testing of ESSENCE and SAS, and technical assistance and training for transitioning jurisdictions.

\section{Keywords}

ESSENCE; Pilot Project; BioSense Platform

\section{Acknowledgments}

Michael Coletta, Roseanne English, Alan Davis, David Walker, Peter Hicks, Alejandro Perez, Paul McMurray, Miguel Torres-Urquidy, Julie Zajac-Cox, Aaron Kite-Powell, Caleb Weideman, Matthew Schwei, Carla Winston, Martha Sanchez, Amanda Wahnich, Katie Arends, Harold Gil, Atar Baer, Yushien Chen

\section{References}

[1] Business Analytics and Business Intelligence Software. SAS. [Internet]. Cary (NC). [cited 2015 Jul 27]. Available from: http:// www.sas.com/

[1] Lombardo, J, Burkom, H, Pavlin, J. ESSENCE II and the Framework for Evaluating Surveillance Systems. MMWR. 2004 Sept 24; 53(Suppl):159-65

\section{${ }^{*}$ Cassandra N. Davis}

E-mail: vts4@cdc.gov 